\title{
EVOLUCIÓN DE LA RESISTENCIA A ANTIBIÓTICOS DE ESCHERICHIA COLI EN MUESTRAS DE ORINA PROCEDENTES DE LA COMUNIDAD
}

\author{
José María Sánchez Merino, Cristina Guillán Maquieiral, Carlos Fuster Foz², Ramiro López
} Medrano², Marta González Pérez ${ }^{3}$, Carmen Raya Fernández² y Jesús García Alonso.

Servicios de Urología, Ginecología', Microbiologíaª y Medicina Preventiva3. Hospital el Bierzo. Ponferrada. León. España.

\begin{abstract}
Resumen.- OBJETIVO: Los objetivos de este trabajo son dos: primero, evaluar la resistencia a varios antibióticos y las tendencias de la misma en un periodo de seis años en cepas de Escherichia coli aisladas en muestras de orina de pacientes atendidos en Atención Primaria en nuestro medio y, segundo, valorar si las pautas de tratamiento empírico comúnmente aceptadas en nuestro país serían aplicables en nuestro entorno en función de los resultados del estudio.
\end{abstract}

MÉTODOS: Se analizaron los urocultivos positivos para Escherichia coli obtenidos de muestras enviadas desde los diez centros de Atención Primaria del Área Sanitaria de El Bierzo y Laciana (León) entre los años 2002 y Rúa da Zanfona, 3 San Pedro de Nos-Oleiros 15176 A Coruña. (España). sanchezuro@hotmail.com
2007. Se determinó la resistencia in vitro de este germen a diversos antibióticos de uso frecuente: fosfomicina, nitrofurantoína, tobramicina, cefuroxima, cefixima, amoxicilina-clavulánico, cotrimoxazol, ciprofloxacino, norfloxacino y ampicilina. Se analizó mediante Chi cuadrado la existencia de diferencias estadísticamente significativas $(p<0,05)$ de sensibilidad, comparando los años 2002 y 2007 para todos los antimicrobianos excepto para cefixima. En este último caso se compararon los resultados obtenidos en 2002 y 2005.

RESULTADOS: Se ha producido un aumento de la resistencia de los aislamientos urinarios de Escherichia coli a todos los antimicrobianos estudiados, menos para la nitrofurantoína, que fue estadísticamente significativo en la mayoría de los casos. Aún así, la resistencia frente a fosfomicina y nitrofurantoína se ha mantenido por debajo del $6 \%$ a lo largo del periodo de estudio. Para tobramicina y cefuroxima apenas ha superado el 10\% y para cefixima se encuentra por debajo del 3,4\%, aunque en este último caso sólo se dispone de datos hasta 2005 en nuestro estudio. La resistencia frente a amoxicilina-clavulánico, inicialmente baja, ha ido aumentando progresivamente hasta alcanzar el 20,6\% en 2007. Lo mismo ocurre para cotrimoxazol, ciprofloxacino, norfloxacino y ampicilina, hasta superar el 32\% en 2007 en el caso de los tres primeros y el $62 \%$ en el último.

CONCLUSIONES: La variación en los patrones de resistencia bacteriana de Escherichia coli obliga a disponer de un conocimiento actualizado de los mismos para adaptar las pautas generales de tratamiento empírico a cada área de salud concreta.

Palabras clave: Infección urinaria. Infección extrahospitalaria. Resistencia microbiana. Tratamiento médico. 
Summary.- OBJECTIVES: The objectives of this work are two: first, to evaluate the resistance of Escherichia coli to several antibiotics and their trends over a six-year period in strands isolated in urine samples from patients receiving health-care in general practitioner offices in our environment; and second, to evaluate if empirical treatment regimens commonly accepted in our country would be applicable in our environment depending on the results of this study.

METHODS: We analyzed the urine cultures positive for Escherichia coli obtained from samples collected at the 10 primary health care centers of the health-care area of El Bierzo and Laciana (Leon, Spain) between the years 2002 and 2007. In vitro resistances of these germs to several common use antibiotics were determined: fosfomycin, nitrofurantoin, tobramycin, cefuroxime, cefixime, amoxicillin-clavulanic acid, cotrimoxazole, ciprofloxacin, norfloxacin, and ampicillin. The existence of statistically significant ( $p<0.05)$ differences in sensitivity, comparing the years 2002 and 2007, including all antimicrobials except cefixime, was analyzed by the chi-square test. For cefixime we compared the results between 2002 and 2005.

RESULTS: An increase of the resistance of Escherichia coli isolated in urine to all antimicrobials under study has occurred, except for nitrofurantoin, being the differences statistically significant in most cases. Nevertheless, resistances to fosfomycin and nitrofurantoin have remained below $6 \%$ throughout the study period. Resistances to tobramycin and cefuroxime were slightly over 10\% and cefixime below $3.4 \%$, although in the last one we only have data until 2005. Resistances to amoxicillinclavulanic acid, initially low, have progressively increase reaching $20.6 \%$ in 2007 . The same has happened for cotrimoxazole, ciprofloxacin, norfloxacin and ampicillin, passing $32 \%$ in 2007 in the first three cases and $62 \%$ in the last one.

CONCLUSIONS: Variations in bacterial resistance patterns for Escherichia coli obliges to have an updated knowledge of them to adapt general empirical treatment uses to each specific health-care area.

Keywords: Urinary tract infection. Community-based infection. Microbial resistance. Medical treatment.

\section{INTRODUCCIÓN}

España es uno de los países europeos más afectados por el aumento de la resistencia a antimicrobianos en algunos de los patógenos bacterianos más frecuentes causantes de patologías en el ser humano, constituyendo la vigilancia epidemiológica una de las principales armas para el control de este problema. Además, aquélla proporciona los datos necesarios para diseñar protocolos de tratamiento empírico individualizados en función de la situación epidemiológica de cada área geográfica (1). En relación con este aspecto, incluso médicos con una magnífica formación carecen a menudo de información actualizada de los patrones de resistencia bacteriana en su área geográfica, necesaria para tomar las decisiones de prescripción adecuadas (2).

Considerando que Escherichia coli es la causa más frecuente de infección urinaria en el medio extrahospitalario $(3,4)$, nuestros objetivos son dos: primero, evaluar la resistencia a varios antibióticos y las tendencias de la misma en cepas de esta bacteria aisladas en muestras de orina de pacientes valorados en Atención Primaria en nuestro medio a lo largo de un periodo de seis años $y$, segundo, valorar si algunas de las pautas de tratamiento empírico comúnmente aceptadas en nuestro país serían aplicables en nuestro entorno en función de los resultados del estudio.

\section{MATERIAL Y MÉTODOS}

Se trata de un estudio epidemiológico descriptivo retrospectivo en el que se analizaron los urocultivos positivos para Escherichia coli obtenidos de muestras enviadas desde los diez centros de Atención Primaria del Área Sanitaria de El Bierzo y Laciana (León) entre los años 2002 y 2007, que en su conjunto prestan atención médica a una población de aproximadamente 150.000 habitantes.

Durante el periodo de estudio se recibieron 38.676 muestras de orina procedentes de Atención Primaria en el laboratorio de microbiología del hospital de referencia del área sanitaria motivo de estudio, que se distribuyeron de la siguiente manera: 6.402 muestras recibidas en el año 2002, 6.889 en 2003, 7.109 en 2004, 6.702 en 2005, 5.662 en 2006 y 5.912 muestras en el año 2007 . Fueron positivas el $19,9 \%$ de las mismas en el año 2002 , el $19,1 \%$ en 2003 , el $19,4 \%$ en 2004 , el $20,6 \%$ en 2005 , el $20 \%$ en 2006 y el $20,2 \%$ en el año 2007 . De forma global fueron positivas el $19,9 \%$ de las muestras analizadas.

Escherichia coli ha sido la bacteria más frecuentemente aislada en orina durante estos años, suponiendo entre el 56 y el $58 \%$ del total de cepas aisladas en Atención Primaria en nuestra área sanitaria. El segundo germen en frecuencia, Enterococcus faecalis, no ha supuesto en ningún momento más del $11 \%$ del total de los aislamientos urinarios. 
Se determinó la sensibilidad in vitro de Escherichia coli a diversos antibióticos de uso frecuente: fosfomicina, nitrofurantoína, tobramicina, las cefalosporinas de segunda generación cefuroxima y la de tercera cefixima, amoxicilina-clavulánico, sulfametoxazol-trimetoprim (cotrimoxazol), las quinolonas ciprofloxacino y norfloxacino y, por último, la ampicilina. Para el análisis estadístico las cepas de sensibilidad intermedia, siendo un porcentaje desdeñable, se incluyeron dentro del grupo de resistentes. Para cefixima sólo dispusimos de datos hasta 2005.

Se utilizó el medio de cultivo CLED y se determinaron las concentraciones mínimas inhibitorias mediante el sistema MicroScan ${ }^{\circledR}$ de Dade Behring. Para la interpretación de la sensibilidad entre los años 2002 a 2005 se aplicaron los criterios del NCCLS (National Committe for Clinical Laboratory Standards) (5) y CLSI (Clinical and Laboratory Standards Institute) en 2006 y 2007 (6). Se analizó mediante Chi cuadrado, bivariado, sin estratificación por edad o sexo (programa RSIGMA para MSDOS) la existencia o no de diferencias estadísticamente significativas $(p<0,05)$ de resistencia, comparando los años 2002 y 2007 . En el caso de la cefalosporina cefixima se compararon los años 2002 y 2005.

\section{RESULTADOS}

La Tabla I muestra la evolución del patrón de resistencia bacteriana de Escherichia coli entre los años 2002 y 2007 , con los valores expresados en porcentaje. Se han remarcado en gris aquellos valores que superan el $20 \%$, que es la cifra por encima de la cual no se recomienda un antimicrobiano para su uso empírico (7). Los datos obtenidos se basaron exclusivamente en hallazgos microbiológicos, sin tener en cuenta criterios clínicos.

Como se observa en la Tabla, se ha producido un aumento de la resistencia a lo largo del periodo de estudio para fosfomicina, tobramicina, cefuroxima, amoxicilina-clavulánico, ciprofloxacino, norfloxacino y ampicilina, en todos los casos estadísticamente significativo $(p<0,01)$. Para cefixima y cotrimoxazol se ha producido igualmente un aumento de las resistencias, pero sin significación estadística. Ha mejorado la resistencia para nitrofurantoína, aunque no es estadísticamente significativa.

Aunque ha habido un aumento global de la resistencia bacteriana de cepas de Escherichia coli aisladas en las muestras de orina procedentes

TABLA I. EVOLUCIÓN DE LA RESISTENCIA BACTERIANA (\%) EN CEPAS DE ESCHERICHIA COLI AISLADAS EN MUESTRAS DE ORINA ENVIADAS POR ATENCIÓN PRIMARIA EN LA COMARCA DEL BIERZO Y LACIANA (LEÓN) ENTRE LOS AÑOS 2002 Y 2007.

\begin{tabular}{|l|c|c|c|c|c|c|}
\hline & $\mathbf{2 0 0 2}$ & $\mathbf{2 0 0 3}$ & $\mathbf{2 0 0 4}$ & $\mathbf{2 0 0 5}$ & $\mathbf{2 0 0 6}$ & $\mathbf{2 0 0 7}$ \\
\hline Fosfomicina & 0,8 & 0,4 & 1 & 1,6 & 2,3 & 2,7 \\
\hline Nitrofurantoína & 5,5 & 5,3 & 3,4 & 5,1 & 2,9 & 3,8 \\
\hline Cefixima & 1,7 & 3,3 & 3,1 & 3,4 & - & - \\
\hline Tobramicina & 6,3 & 6,2 & 5,7 & 6,2 & 10,1 & 9,7 \\
\hline Cefuroxima & 3,5 & 5 & 4,2 & 6,6 & 8,4 & 10,1 \\
\hline Amoxicilina -clavulánico & 6,9 & 6,2 & 6,5 & 12,6 & 17,1 & 20,6 \\
\hline Cotrimoxazol* & 28,5 & 25,1 & 28 & 28,8 & 30,5 & 32,4 \\
\hline Ciprofloxacino & 22,9 & 21,6 & 23,7 & 24,8 & 30,2 & 32,5 \\
\hline Norfloxacino & 24,2 & 21,8 & 24,1 & 24,7 & 30 & 32,9 \\
\hline Ampicilina & 56 & 52,2 & 55,4 & 54 & 53,5 & 62,6 \\
\hline
\end{tabular}

Valores expresados en porcentaje. * Sulfametoxazol-trimetoprima.

-: Dato no disponible. 
de Atención Primaria en nuestro medio a los antimicrobianos estudiados, fosfomicina y nitrofurantoína mantienen un bajo perfil de resistencia, así como la cefixima, la cefuroxima y los aminoglucósidos. Aunque sólo se reflejan en la tabla los datos correspondientes a la tobramicina, la gentamicina presenta porcentajes de resistencia casi idénticos en nuestro medio. La amoxicilina-clavulánico comenzaría a no ser recomendable para el tratamiento empírico de la infección urinaria en el ámbito extrahospitalario en nuestra área de salud a diferencia de años anteriores y se encontrarían en valores inaceptables de resistencia para el uso antes mencionado el cotrimoxazol, ciprofloxacino, norfloxacino y ampicilina.

\section{DISCUSIÓN}

El incremento del consumo de antibióticos es una de las principales causas reconocidas del aumento de las resistencias bacterianas a los mismos (8). En este sentido, España fue el quinto país en ventas de quinolonas de la Unión Europea en el año 2002 (9). No en vano las cifras de resistencia a quinolonas en nuestro país superan a las de otros países europeos (10).

Haciendo hincapié en este mismo aspecto, la presidencia de Health Consumer Powerhouse en la presentación del Índice de Consumidores de la Sanidad Europea 2007 (EHCI) (11), incidió en el hecho de que España "tiene un alto índice de infecciones resistentes a los antibióticos" y a pesar de reconocer que "la posibilidad de adquirir cualquier tipo de antibióticos sin receta médica [aunque en teoría no es así y las farmacias sólo deben facilitar el antibiótico si se acude con receta] puede parecer atractiva desde el punto de vista del consumidor", insistió no obstante en que "la propagación de infecciones mortales es un precio muy alto que se paga por ello" (sic) (12). Algún otro estudio sugiere que es probable que en realidad España esté a la cabeza del consumo de antibióticos en el ámbito extrahospitalario en Europa si a los datos obtenidos de las prescripciones con cargo al Sistema Nacional de Salud se suman la dispensación sin receta, la automedicación y la prescripción privada (8).

También se ha implicado en esta elevada tasa de resistencias la ingesta de pequeñas dosis de antimicrobianos dentro de la cadena alimentaria por consumo de carne procedente de animales tratados con antibióticos (13).

Teniendo en cuenta la presión selectiva que ejerce el elevado consumo de antibióticos en nuestro entorno, es necesario actualizar los datos relativos a los patrones de resistencia bacteriana, ya que éstos pueden variar entre distintas zonas e incluso en una misma área geográfica con el paso del tiempo. Así, en nuestro caso concreto, en 1998 las cepas de Escherichia coli resistentes a ciprofloxacino y norfloxacino eran algo inferiores al $20 \%$ (3). Es decir, aún era factible su uso para el tratamiento empírico. Sirva también como ejemplo el llamativo incremento de la resistencia de Escherichia coli frente a amoxicilinaclavulánico, que ha pasado de un $6,9 \%$ en 2002 a un $20,6 \%$ en 2007.

La principal desventaja de nuestro estudio de la resistencia bacteriana de Escherichia coli en muestras de orina es que no tiene en cuenta las características clínicas de los pacientes. Los urocultivos proceden de población urbana y rural extrahospitalaria con diagnóstico de sospecha de infección urinaria o control postratamiento, pero no disponemos de datos estratificados por edad, sexo, tipo de patología y la existencia o no de tratamiento antibiótico previo. Sin embargo, nuestro estudio se limita al estudio de la resistencia bacteriana para Escherichia coli, el germen causal más frecuentemente aislado en las infecciones urinarias que pueden tratar los médicos de Atención Primaria. En relación con este aspecto, todas las guías de recomendación para el tratamiento empírico de las infecciones urinarias habitualmente abordadas en el ámbito extrahospitalario no especializado se basan en la sensibilidad de la bacteria más frecuentemente aislada, es decir, la Escherichia coli. Por otra parte, las propias guías de terapéutica antimicrobiana insisten en utilizar los datos proporcionados por los laboratorios de microbiología como orientación para el tratamiento empírico de las infecciones urinarias. Pero, de cualquier manera, la mejor aproximación se obtendría estratificando los resultados por edad, sexo y tipo de patología.

Teniendo en consideración todo lo anterior, tendríamos que adaptar las recomendaciones de las pautas de tratamiento empírico de la infección urinaria en el medio extrahospitalario a la realidad de nuestro entorno epidemiológico $(14,15)$. Si nos referimos a la patología a la que más frecuentemente se puede enfrentar el médico de Atención Primaria, esto es, la cistitis simple o no complicada, la guía de práctica clínica promovida por la Asociación Española de Urología (14) recomienda como tratamiento de primera elección la fosfomicina trometamol, como de segunda elección sulfametoxazol-trimetoprim, norfloxaciono, ciprofloxaciono, amoxicilina-clavulánico y cefixima, y como de tercera la nitrofurantoína. Otra guía de terapéutica antimicrobiana bien conocida (15) recomienda para el tratamiento antimicrobiano empírico de la cistitis simple la cefuroxima, cefixima, una fluorquinolona, amoxicilina-clavulánico, fosfomicina trometamol y nitrofurantoína. De esta manera, en nuestro medio concreto, objeto de estudio, el tra- 
tamiento de primera elección podría ser, de acuerdo con las guías antes mencionadas, la fosfomicina, cefixima y cefuroxima, dejando como tratamiento de $2^{\underline{a}}$ elección, con cierta reserva en el momento actual, la amoxicilina-clavulánico.

Una posible explicación para el aumento de la resistencia a amoxicilina-clavulánico es el gran uso de este antibiótico en la población infantil atendida en Atención Primaria en nuestro medio, que también está incluida en el estudio.

Por otra parte, no se podría recomendar el cotrimoxazol, norfloxacino y ciprofloxacino para el tratamiento empírico de la infección urinaria en el ámbito extrahospitalario en nuestra área de salud.

Con respecto a los otros antimicrobianos objeto de estudio, la tobramicina presenta una magnífica actividad frente a Escherichia coli, aunque no es de uso habitual en Atención Primaria. Por otra parte, la nitrofurantoína, al igual que la fosfomicina, presenta unos niveles sorprendentemente bajos de resistencias.

\section{CONCLUSIÓN}

Como conclusión, se observa un aumento de la resistencia de los aislamientos urinarios de Escherichia coli a prácticamente todos los antimicrobianos estudiados. Derivado de este hecho, la variación en los patrones de resistencia bacteriana de este germen obliga a disponer de un conocimiento actualizado de los mismos para adaptar las pautas generales de tratamiento empírico a cada área de salud concreta.

Finalmente, siendo importante todo lo anterior, sería necesario tomar las medidas necesarias para el control de la resistencia bacteriana, implicando a todos los agentes que hacen uso de los antibióticos, incluyendo el sector veterinario. Incidiendo en este aspecto, desde el Centro Nacional de Microbiología del Instituto de Salud Carlos III se recomienda actuar con contramedidas si no se quiere volver a una situación semejante a la de la época preantibiótica (sic) (1).

\section{BIBLIOGRAFÍA y LECTURAS RECOMENDADAS ( ${ }^{*}$ lectura de interés $y^{* *}$ lectura fundamental)}

**1. OTEO, J.; CAMPOS, J.: "Valor de los sistemas de vigilancia de resistencia a antibióticos". Enferm. Infecc. Microbiol. Clin., 21: 123, 2003.

**2. CAMPOS, J.; BAQUERO, F.: "Resistencia a antibióticos: ¿Qué hacer ahora?”. Med. Clin. (Barc), 119: 656, 2002.
3. SÁNCHEZ MERINO, J.M.; GUILLÁN MAQUIEIRA, C.; FUSTER, C. y cols.: "Sensibilidad Microbiana de Escherichia coli en Infecciones Urinarias Extrahospitalarias". Actas Urol. Esp., 27: 783, 2003.

4. SÁNCHEZ MERINO, J.M.; GUILLÁN MAQUIEIRA, C.; FUSTER, C. y cols.: "Sensibilidad Microbiana de Escherichia coli en bacteriurias en el área sanitaria del Bierzo en el año 2003". Actas Urol. Esp., 28: 588, 2004.

5. CLINICALAND LABORATORY STANDARDS INSTITUTE / NCCLS.: "Performance Standards for Antimicrobial Susceptibility Testing; Fifteenth Informational Supplement. CLSI / NCCLS document M100-S15 [ISBN 1-56238-556-9]". Clinical and Laboratory Standards Institute, 940 West Valley Road, Suite 1400; Wayne, Pennsylvania 19087-1898, USA,2005.

6. CLINICALAND LABORATORY STANDARDS INSTITUTE.: "Performance Standards for Antimicrobial Susceptibility Testing; Seventeenth Informational Supplement. CLSI document M100-S17 [ISBN 1-56238-625-5]". Clinical and Laboratory Standards Institute, 940 West Valley Road, Suite 1400; Wayne, Pennsylvania 190871898, USA, 2007.

7. GUPTA, K.; SCHOLES, D.; STAMM, WE.: "Increasing prevalence of antimicrobial resistance among uropathogens causing acute uncomplicated cystitis in women". JAMA, 281: 736, 1999.

**8. LÁZARO, E.; OTEO, J.: "Evolución del consumo y de la resistencia a antibióticos en España". Inf. Ter. Sist. Nac. Salud, 30: 10, 2006.

*9. GOOSSENS, H.; FERECH, M.; VANDER, R. y cols.: "Outpatient antibiotic use in Europe and association with resistace: A cross-national database study". Lancet, 365: 579, 2005.

10. KAHLMETER, G.: The ECO-SENS Project: a prospective, multinational, multicentre epidemiological survey of the prevalence and antimicrobial susceptibility of urinary tract pathogens-interim report. J. Antimicrob. Chemother., 46 (Suppl S1): $15,2000$.

11. http://www.healthpowerhouse.com/media/Rapport_EHCI_2007.pdf

12. http://db.doyma.es/cgi-bin/wdbcgi.exe/doyma/ press.plantilla?ident $=51027 \&$ mail $=\mathrm{Si}$

13. EL GLOBAL: "La FDA prohíbe unos antibióticos utilizados en la alimentación de pollos". El global 2001 Enero 19; Sección Productos: 14.

**14. PALOU, J.; MILLÁN, F.: "Guía de práctica clínica. Cistitis no complicada en la mujer". Asociación Española de Urología.

**15. MENSA, J.; GATELL, J.M.; AZANZA, J.R. y cols.: "Guía de Terapéutica Antimicrobiana". $18^{\mathrm{a}}$ ed. Elsevier Doyma, S.L.: Barcelona, 2008. 